\title{
An asymptotic property of the Camassa-Holm equation on the half-line
}

\author{
Jia Jia and Shunguang Kang*
}

"Correspondence:

kangshunguang@163.com

College of Information Engineering,

Tarim University, Alar, 843300

P.R. China

\section{Springer}

\begin{abstract}
The paper addresses the asymptotic properties of Camassa-Holm equation on the half-line. That is, using the method of asymptotic density, under the assumption that it is unique, the paper proves that the positive momentum density of the Camassa-Holm equation is a combination of Dirac measures supported on the positive axis. This means that as time goes to infinity, the momentum density concentrates in small intervals moving right with different constant speeds.
\end{abstract}

MSC: $35053 ; 37 K 40$

Keywords: Camassa-Holm equation; asymptotic density; momentum density; asymptotic property; Dirac measures

\section{Introduction}

In this paper we consider the following initial boundary value problem of the CamassaHolm equation on the half line:

$$
\left\{\begin{array}{l}
u_{t}-u_{t x x}+3 u u_{x}=2 u_{x} u_{x x}+u u_{x x x}, \quad t>0, x \in \mathbb{R}_{+}, \\
u(0, x)=u_{0}(x), \quad x \in \mathbb{R}_{+}, \\
u_{x}^{(2 k)}(t, 0)=u_{x}^{(2 k-2)}(t, 0)=\cdots=u(t, 0)=0, \quad t \geq 0,
\end{array}\right.
$$

where $u_{0}^{(2 k)}(0)=u_{0}^{(2 k-2)}(0)=\cdots=u_{0}(0)$. Let $h=u-u_{x x}$ be the momentum density. The Camassa-Holm equation on the half line in momentum density form is

$$
\left\{\begin{array}{l}
h_{t}+u h_{x}+2 u_{x} h=0, \quad t>0, x \in \mathbb{R}_{+}, \\
h(t, x)=\left(1-\partial_{x}^{2}\right) u(t, x), \quad t>0, x \in \mathbb{R}_{+}, \\
h(x, 0)=h_{0}(x)=\left(1-\partial_{x}^{2}\right) u_{0}, \quad x \in \mathbb{R}_{+}, \\
\partial_{x}^{2 k-2} h(t, 0)=\cdots=\partial_{x}^{2} h(t, 0)=h(t, 0)=0, \quad t \geq 0 .
\end{array}\right.
$$

The Camassa-Holm equation is a model for the unidirectional propagation of shallow water waves over a flat bottom. It has a bi-Hamiltonian structure [1] and is completely integrable [2, 3]. Its solitary waves are peaked [4]. The convergence of the solution of the Camassa-Holm equation to the distributional solution of the Burgers one and the solution of the dispersive equation converging to the unique entropy solution of a scalar conservation law are proven $[5,6]$. In [4], numerical studies illustrate that some nonnegative initial condition evolves into a train of peakons moving with different velocities. On the

(c) The Author(s) 2016. This article is distributed under the terms of the Creative Commons Attribution 4.0 International License (http://creativecommons.org/licenses/by/4.0/), which permits unrestricted use, distribution, and reproduction in any medium, provided you give appropriate credit to the original author(s) and the source, provide a link to the Creative Commons license, and indicate if changes were made. 
theoretical side of the topic, there is research on the stability of peakons, which says that at least for initial values close to peakons, it will stay close to the peakons. The shapes of peakons and multipeakon/antipeakons are stable under small perturbations, making them recognizable physically [7-9]. Constantin and Strauss [7] proved the stability of a single peakon among $C\left([0 ; T] ; H^{1}(R)\right)$ solutions. El Dika and Molinet proved the stability of multi-peakons [8] and multi-anti-peakons-peakons [9] among a slightly more regular class of solutions.

In this article, we use the method of asymptotic density of the momentum to show that under the assumption of its uniqueness, at the momentum level, the solution approaches a train of peakons moving right with different speeds. This approach was introduced by Chen and Frid [10] to study the asymptotic behavior of the entropy solutions of conservation laws. It has been used to discuss the asymptotic behavior of the vorticity of the two dimensional incompressible Euler equation by Iftimie, Lopes, and Nussenzveig [11, 12]. Reference [13] is an exposition. Notice that the vorticity in the incompressible Euler equations and the momentum density in the Camassa-Holm equation are similar. They satisfy similar first order nonlinear nonlocal equations and give the velocity through similar integrals. There are also differences. The 2D Euler flow preserves volume and the vorticity is transported along the particle trajectories, but the same do not hold for the Camassa-Holm flow and its momentum density. We have studied the asymptotic property for a global strong solutions of the Camassa-Holm equation by the approach in [14], and the asymptotic property of the solutions of the Degasperis-Procesi equation is studied [15]. Results on the half-line may not automatically follow those on the whole line. For example, if the momentum density is non-positive, the solution on $\mathbb{R}$ approaches a left moving peakon train whereas on $\mathbb{R}_{+}$, solutions with non-positive initial momentum densities must blow up in finite time (see [16]) and we cannot discuss their asymptotic properties. New, we proceed to a study of the momentum density of the Camassa-Holm equation on the half line.

In this paper, we will investigate initial boundary value problems of the Camassa-Holm equation with initial data $u_{0} \in H^{s}\left(\mathbb{R}_{+}\right) \cap H_{0}^{1}\left(\mathbb{R}_{+}\right), s>\frac{5}{2}$, where $\mathbb{R}_{+}=[0, \infty)$. Let $k \in \mathbb{N} \backslash\{0\}$, and for $2 k+\frac{1}{2}<s \leq 2 k+2$, we set

$$
G^{S}\left(\mathbb{R}_{+}\right)=\left\{u \in H^{S}\left(\mathbb{R}_{+}\right) \mid u^{(2 k)}(0)=u^{(2 k-2)}(0)=\cdots=u(0)\right\}
$$

Theorem 1.1 ([16], Theorem 2.2) Let $u_{0} \in G^{s}\left(\mathbb{R}_{+}\right)$, with $k \in \mathbb{N} \backslash\{0\}$, and $2 k+\frac{1}{2}<s \leq 2 k+2$. Assume that $h_{0}(x):=u_{0}(x)-u_{0, x x}(x) \geq 0$ for all $x \in \mathbb{R}_{+}$. Then there exists a global solution $u(t, x)$ to (1.1) such that, for all $T>0$,

$$
u=u\left(\cdot, u_{0}\right) \in C\left([0, T) ; G^{s}\left(\mathbb{R}_{+}\right)\right) \cap C^{1}\left([0, T) ; G^{s-1}\left(\mathbb{R}_{+}\right)\right) .
$$

Moreover, the solution depends continuously on the initial data, i.e., the mapping $u_{0} \longmapsto$ $u\left(\cdot, u_{0}\right): G^{s}\left(\mathbb{R}_{+}\right) \longrightarrow C\left([0, T) ; G^{s}\left(\mathbb{R}_{+}\right)\right) \cap C^{1}\left([0, T) ; G^{s-1}\left(\mathbb{R}_{+}\right)\right)$is continuous.

Definition 1.2 Let $u$ be a global solution of (1.1), and the initial momentum density $h_{0} \geq 0$ is compactly supported. For $t>0$, let

$$
\tilde{h}(t, y):=\operatorname{th}(t, t y)
$$

be the scaled momentum density of $u$. 
Definition 1.3 Let $[a, b] \subset \mathbb{R}_{+}$be a finite interval, and $\operatorname{supp} \tilde{h}(t, \cdot) \subset[a, b]$ for all $t \geq 1$. Suppose there is a sequence $t_{k} \rightarrow \infty$ as $k \rightarrow \infty$, and a positive Radon measure $\mu \in \mathcal{M}[a, b]$ such that

$$
\tilde{h}\left(t_{k}, \cdot\right) \rightarrow \mu, \quad \text { as } k \rightarrow \infty .
$$

Then we call $\mu$ an asymptotic density associated with the initial momentum density $h_{0}$.

\section{Remark 1.4}

(a) $\mathcal{M}[a, b]$ is the space of regular Borel measures on $[a, b]$.

(b) The convergence is the weak-* convergence in $\mathcal{M}[a, b]$, i.e. for all $\psi \in C[a, b]$, $\int_{a}^{b} \tilde{h}(t, y) \psi(y) \mathrm{d} y \rightarrow\langle\mu, \psi\rangle$.

(c) The asymptotic densities associated with $h_{0}$ may not be unique.

The following is the main result of this article.

Theorem 1.5 Let $u$ be a global solution of $(1.1)$, and $h_{0}(\cdot)=h(0, \cdot) \geq 0$ has compact support. For $t \geq 0$, suppose that $\tilde{h}(t, \cdot)$ has a unique asymptotic density $\mu$ associated with $h_{0}$. Then there exist finitely or countably infinitely many $m_{i}, \alpha_{i} \in[0, \infty)$ such that

$$
\mu=\sum_{i=1}^{\infty} m_{i} \delta_{\alpha_{i}}
$$

where $\delta_{\alpha_{i}}$ is the delta function supported at $\alpha_{i}$, and

(a) $\alpha_{i} \leq \frac{3}{2} m_{i}$, for all $i$.

(b) $\alpha_{i} \in[0, M)$, for any $i$, where $M=\|u\|_{L^{\infty}\left(\mathbb{R}_{+} \times \mathbb{R}_{+}\right)}$.

(c) $\alpha_{i} \rightarrow 0$ as $i \rightarrow \infty$ and $\alpha_{i} \neq \alpha_{j}$ if $i \neq j$.

(d) $m_{i}>0$ and $\sum_{i=1}^{\infty} m_{i}=\left\|h_{0}\right\|_{L^{1}\left(\mathbb{R}_{+}\right)}$.

In other words, the momentum densities of such global solutions concentrates in slumps moving right approaching different speeds.

\section{Preliminaries}

Consider the following differential equation:

$$
\left\{\begin{array}{l}
\frac{\mathrm{d} q(t, x)}{\mathrm{d} t}=u(t, q(t, x)), \quad t>0, x \in \mathbb{R}_{+} \\
q(0, x)=x, \quad x \in \mathbb{R}_{+} .
\end{array}\right.
$$

Applying classical results in the theory of ordinary differential equations, one can obtain the following results on $q$.

Lemma 2.1 Let $u \in C\left([0, T) ; G^{s}\left(\mathbb{R}_{+}\right)\right) \cap C^{1}\left([0, T) ; G^{s-1}\left(\mathbb{R}_{+}\right)\right)$be a nonnegative solution of (1.1) for all $T>0$, Then the (2.1) has a unique solution $q \in C^{1}\left([0, T) \times \mathbb{R}_{+}, \mathbb{R}_{+}\right)$. Moreover, the map $q(t, \cdot)$ is an increasing diffeomorphism of $\mathbb{R}_{+}$. And

$$
h(t, q(t, x)) q_{x}^{3}(t, x)=h_{0}(x), \quad(t, x) \in\left([0, T) \times \mathbb{R}_{+}\right) .
$$




\section{Remark 2.2}

(a) If supp $h_{0} \subset[a, b]$, then, for all $t \in(0, T)$, supp $h(t, \cdot) \subset[q(t, a), q(t, b)]$.

(b) If $h_{0} \geq 0$, then, for $t \in[0, T), h(t, \cdot) \geq 0$.

Lemma 2.3 Let $u$ be a global solution of (1.1). Suppose that $h_{0} \in L^{1}\left(\mathbb{R}_{+}\right)$and $h_{0} \geq 0$. Then, for $t \geq 0$, we have

$$
\|\tilde{h}(t, \cdot)\|_{L^{1}\left(\mathbb{R}_{+}\right)}=\|h(t, \cdot)\|_{L^{1}\left(\mathbb{R}_{+}\right)}=\left\|h_{0}(t, \cdot)\right\|_{L^{1}\left(\mathbb{R}_{+}\right)} \cdot
$$

Proof From $h_{0} \geq 0$ and Remark 2.2(b) $h(t, \cdot) \geq 0$, for $t>0$, the first equality of (2.2) results from a change of variable. The seconde equality can be got from (1.2) using integration by parts. That is,

$$
\frac{\mathrm{d}}{\mathrm{d} t} \int_{\mathbb{R}_{+}} h(t, x) \mathrm{d} x=\int_{\mathbb{R}_{+}}\left(-u h_{x}-2 u_{x} h\right) \mathrm{d} x=\int_{\mathbb{R}_{+}}-u_{x} h \mathrm{~d} x=0 .
$$

Proposition 2.4 Let $u$ is a global solution of (1.1), and $h$ is the momentum density of $u$. Then

$$
u(t, x)=\frac{1}{2} \int_{\mathbb{R}_{+}}\left(\mathrm{e}^{-|x-y|}-\mathrm{e}^{-|x+y|}\right) h(t, y) \mathrm{d} y .
$$

Proof Let

$$
\begin{aligned}
& \bar{h}(t, x)= \begin{cases}h(t, x), & x \geq 0, \\
-h(t,-x), & x<0,\end{cases} \\
& \bar{u}(t, x)= \begin{cases}u(t, x), & x \geq 0, \\
-u(t,-x), & x<0 .\end{cases}
\end{aligned}
$$

For convenience, the following proof omits the $t$. When $x<0$,

$$
\bar{u}^{\prime \prime}(x)=\frac{\mathrm{d}^{2}}{\mathrm{~d} x^{2}} \bar{u}(x)=\frac{\mathrm{d}^{2}}{\mathrm{~d} x^{2}}[-u(-x)]=-u^{\prime \prime}(-x)
$$

Then

$$
\bar{u}(x)-\bar{u}^{\prime \prime}(x)=-u(-x)+u^{\prime \prime}(-x)=-\left[u(-x)-u^{\prime \prime}(-x)\right]=-h(-x)=\bar{h}(x) .
$$

So $\bar{h}(t, x)$ is the momentum density of $\bar{u}(t, x)$ on the whole line. Then

$$
\begin{aligned}
\bar{u}(x) & =\frac{1}{2} \int_{\mathbb{R}} \mathrm{e}^{-|x-y|} \bar{h}(y) \mathrm{d} y=\frac{1}{2} \int_{\mathbb{R}_{+}} \mathrm{e}^{-|x-y|} \bar{h}(y) \mathrm{d} y+\frac{1}{2} \int_{\mathbb{R}_{-}} \mathrm{e}^{-|x-y|} \bar{h}(y) \mathrm{d} y \\
& =I+I I .
\end{aligned}
$$

Because

$$
I=\frac{1}{2} \int_{\mathbb{R}_{+}} \mathrm{e}^{-|x-y|} \bar{h}(y) \mathrm{d} y=\frac{1}{2} \int_{\mathbb{R}_{+}} \mathrm{e}^{-|x-y|} h(y) \mathrm{d} y
$$


and

$$
\begin{aligned}
I I & =\frac{1}{2} \int_{\mathbb{R}_{-}} \mathrm{e}^{-|x-y|} \bar{h}(y) \mathrm{d} y=\frac{1}{2} \int_{\mathbb{R}_{-}} \mathrm{e}^{-|x-y|}(-h(-y)) \mathrm{d} y \\
& =-\frac{1}{2} \int_{\mathbb{R}_{+}} \mathrm{e}^{-|x+z|} h(z) \mathrm{d} z,
\end{aligned}
$$

we have

$$
\bar{u}(x)=I+I I=\frac{1}{2} \int_{\mathbb{R}_{+}}\left(e^{-|x-y|}-e^{-|x+y|}\right) h(t, y) \mathrm{d} y .
$$

We record here two formulas frequently used later. From (2.3), we get

$$
u(t, t x)=\frac{1}{2} \int_{\mathbb{R}_{+}}\left(e^{-|t x-y|}-e^{-|t x+y|}\right) h(t, y) \mathrm{d} y=\frac{1}{2} \int_{\mathbb{R}_{+}}\left(e^{-t|x-z|}-e^{-t|x+z|}\right) \tilde{h}(t, z) \mathrm{d} z
$$

Differentiate (2.3) with respect to the spatial variable to get

$$
\begin{aligned}
u_{x}(t, x) & =\frac{1}{2} \int_{\mathbb{R}_{+}}\left(\operatorname{sgn}(y-x) e^{-|x-y|}+e^{-|x+y|}\right) h(t, y) \mathrm{d} y \\
& =\frac{1}{2} \int_{\mathbb{R}_{+}}\left(\operatorname{sgn}(t z-x) e^{-|x-t z|}+e^{-|x+t z|}\right) \tilde{h}(t, z) \mathrm{d} z .
\end{aligned}
$$

Hence

$$
u_{x}(t, t x)=\frac{1}{2} \int_{\mathbb{R}_{+}}\left(\operatorname{sgn}(z-x) e^{-t|x-z|}+e^{-t|x+z|}\right) \widetilde{h}(t, z) \mathrm{d} z
$$

Lemma 2.5 Let $u$ be a global solution of (1.1). Suppose that $h_{0} \geq 0$ and $\operatorname{supp}\left(h_{0}\right) \subset[a, b] \subset$ $\mathbb{R}_{+}$. Then, for $t \geq 1$,

$$
\operatorname{supp} \tilde{h}(t, \cdot) \subset\left[\frac{a}{t}, \frac{b}{t}+M\right]
$$

Here $M:=\|u\|_{L^{\infty}\left(\mathbb{R}_{+} \times \mathbb{R}_{+}\right)}$. There exists $d>M$, such that, for $t \geq 1, \operatorname{supp} \tilde{h}(t, \cdot) \subset[0, d]$.

Proof Notice that $u_{0} \in G^{s}\left(\mathbb{R}_{+}\right)$, with $k \in \mathbb{N} \backslash\{0\}$, and $2 k+\frac{1}{2}<s \leq 2 k+2$, implies that $h_{0} \in H^{s-2}\left(\mathbb{R}_{+}\right) \subset L^{\infty}\left(\mathbb{R}_{+}\right)$, and that the $h_{0}$ has compact support implies that $h_{0} \in L^{1}\left(\mathbb{R}_{+}\right)$. From (2.6) and the Remark 2.2(b), $h(t, \cdot) \geq 0$ for all $t \geq 0$. Equation (2.3) and Lemma 2.3 imply that, for all $(t, x) \in[0, \infty) \times \mathbb{R}_{+}$,

$$
0 \leq u(t, x) \leq \frac{1}{2}\|h(t, \cdot)\|_{L^{1}\left(\mathbb{R}_{+}\right)}=\frac{1}{2}\left\|h_{0}\right\|_{L^{1}\left(\mathbb{R}_{+}\right)}<\infty
$$

Let $M:=\|u\|_{L^{\infty}\left(\mathbb{R}_{+} \times \mathbb{R}_{+}\right)}$. By the Remark 2.2(a), supp $h(t, \cdot) \subset[a, b+M t]$, then

$$
\operatorname{supp} \tilde{h}(t, \cdot) \subset\left[\frac{a}{t}, \frac{b}{t}+M\right]
$$


Lemma $2.6([13,17])$ Let $\mu \in \mathcal{M}[c, d]$ be a nonnegative measure. Then there exist a nonnegative continuous measure $v \in \mathcal{M}[c, d]$, countably (including finitely) many real numbers $m_{i}>0$, and $\alpha_{i} \in[c, d]$ such that

$$
\mu=v+\Sigma_{i} m_{i} \delta_{\alpha_{i}}
$$

In particular, given $\varepsilon>0$, there exists $\delta>0$ such that, for an interval $I \subset[c, d]$ with $|I|<$ $\delta, v(I)<\varepsilon$.

\section{Proof of the main theorem}

We prove Theorem 1.5 in this section. We will obtain information on the asymptotic density $\mu$ by testing it with $\varphi \in C_{c}\left(\mathbb{R}_{+}\right)$.

Proposition 3.1 Suppose that $h$ satisfies (1.2). Then $\widetilde{h}$ satisfies

$$
\frac{\partial}{\partial t} \tilde{h}(t, y)-\frac{\partial}{\partial y}\left[\frac{y \tilde{h}(t, y)}{t}\right]+\frac{u(t, t y)}{t} \frac{\partial}{\partial y} \tilde{h}(t, y)+2 u_{x}(t, t y) \tilde{h}(t, y)=0
$$

Proof Calculate directly to get

$$
\begin{aligned}
& \frac{\partial}{\partial t} \tilde{h}(t, y)=h(t, t y)+t h_{t}(t, t y)+t y h_{x}(t, t y), \\
& -\frac{\partial}{\partial y}\left[\frac{y \tilde{h}(t, y)}{t}\right]=-h(t, t y)-t y h_{x}(t, t y), \\
& \frac{u(t, t y)}{t} \frac{\partial}{\partial y} \tilde{h}(t, y)=t u(t, t y) h_{x}(t, t y), \\
& 2 u_{x}(t, t y) \tilde{h}(t, y)=2 t u_{x}(t, t y) h(t, t y) .
\end{aligned}
$$

From (1.2), we get (3.1).

Proposition 3.2 Suppose $h_{0} \geq 0$ and has compact support. Let $\varphi \in C_{c}\left(\mathbb{R}_{+}\right)$, and $\phi \in$ $C^{1}\left(\mathbb{R}_{+}\right)$be an antiderivative of $\varphi$. Let $t_{k} \rightarrow \infty$ be a sequence of time such that $\tilde{h}\left(t_{k}, \cdot\right) \rightarrow \mu$, an asymptotic density with $\mu=v+\sum_{i} m_{i} \delta_{\alpha_{i}}$, v continuous, $m_{i}>0, \alpha_{i} \in[c, d]$ as given by Lemma 2.6. Then

$$
\begin{aligned}
& \limsup _{k \rightarrow \infty}\left|\int_{\mathbb{R}_{+}} \phi(y) u\left(t_{k}, t_{k} y\right) \frac{\partial}{\partial y}\left[\tilde{h}\left(t_{k}, y\right)\right] \mathrm{d} y+2 \int_{\mathbb{R}_{+}} t_{k} \phi(y) u_{x}\left(t_{k}, t_{k} y\right) \tilde{h}\left(t_{k}, y\right) \mathrm{d} y\right| \\
& \quad \leq \frac{3}{2} \sum_{i}\left|\varphi\left(\alpha_{i}\right)\right| m_{i}^{2} .
\end{aligned}
$$

Proof Fix $\varphi \in C_{c}\left(\mathbb{R}_{+}\right)$. We divide the proof into three steps:

Step 1. Given $\varepsilon>0$, since $\sum_{i=1}^{\infty} m_{i}<\infty$, there exists an $N=N(\varepsilon)$ such that $\sum_{i>N} m_{i}<\frac{\varepsilon}{4}$. Choose $\delta=\delta(\varepsilon)>0$ such that when $|I|<\delta, v(I) \leq \frac{\varepsilon}{4}$ and

$$
\begin{aligned}
& \mu\left(\left[\alpha_{i}-2 \delta, \alpha_{i}+2 \delta\right]\right)<m_{i}(1+\varepsilon), \quad i=1, \ldots, N, \\
& {\left[\alpha_{i}-\delta, \alpha_{i}+\delta\right] \cap\left[\alpha_{j}-\delta, \alpha_{j}+\delta\right]=\emptyset, \quad \text { for } i \neq j, \text { and } 1 \leq i, j \leq N,}
\end{aligned}
$$




$$
\left|\varphi(y)-\varphi\left(\alpha_{i}\right)\right|<\varepsilon, \quad \forall y \in\left[\alpha_{i}-2 \delta, \alpha_{i}+2 \delta\right], i=1, \ldots, N
$$

As $\tilde{h}\left(t_{k}, \cdot\right) \rightarrow \mu$, there is a $K_{0}>0$ such that, for any integer $k>K_{0}$,

$$
\int_{\left[\alpha_{i}-2 \delta, \alpha_{i}+2 \delta\right]} \tilde{h}\left(t_{k}, y\right) \mathrm{d} y<m_{i}(1+\varepsilon), \quad \forall i=1, \ldots, N
$$

and for any interval $I \subset \mathbb{R}_{+} \backslash \bigcup_{i=1}^{N}\left(\alpha_{i}-\frac{1}{2} \delta, \alpha_{i}+\frac{1}{2} \delta\right)$, with $|I|<\delta$, we claim that

$$
\int_{I} \tilde{h}\left(t_{k}, y\right) \mathrm{d} y<\varepsilon
$$

To see this, recall from the first paragraph of step 1 , we get

$$
(\mu-v)(I) \leq \sum_{i>N} m_{i}<\frac{\varepsilon}{4}
$$

then

$$
\mu(I)<\frac{\varepsilon}{2}
$$

Let $\operatorname{supp} \tilde{h}(t, \cdot) \subset J \subset \mathbb{R}_{+}$. Write $J \backslash \bigcup_{i=1}^{N}\left(\alpha_{i}-\frac{1}{2} \delta, \alpha_{i}+\frac{1}{2} \delta\right)$ as $\bigcup_{i=1}^{L} J_{j}$, with $J_{j}$ mutually disjoint and $\left|J_{j}\right| \leq \delta$. From (3.8), we get

$$
\mu\left(J_{j}\right)<\frac{\varepsilon}{2}, \quad \forall j=1, \ldots, L
$$

As $\tilde{h}\left(t_{k}, \cdot\right) \rightarrow \mu$, there is a sufficiently big $K_{0}$, such that (3.6) holds, and

$$
\int_{J_{j}} \tilde{h}\left(t_{k}, y\right) \mathrm{d} y<\frac{\varepsilon}{2}, \quad \forall j=1, \ldots, L, k>K_{0} .
$$

It is obvious that $I$ intersect two $J_{j}$ at most, then from (3.9), we obtain equation (3.7).

Step 2. For $i \in\{1, \ldots, N\}$, Let

$$
E_{i}=\left[\alpha_{i}-\delta, \alpha_{i}+\delta\right], \quad F_{i}=\left[\alpha_{i}-2 \delta, \alpha_{i}+2 \delta\right]
$$

and

$$
E=E_{1} \cup \cdots \cup E_{N} \text {. }
$$

Define

$$
\begin{aligned}
B_{k} & :=\int_{\mathbb{R}_{+}} \phi(y) u\left(t_{k}, t_{k} y\right) \frac{\partial}{\partial y}\left[\tilde{h}\left(t_{k}, y\right)\right] \mathrm{d} y+2 \int_{\mathbb{R}_{+}} \phi(y) u_{x}\left(t_{k}, t_{k} y\right) \tilde{h}\left(t_{k}, y\right) t_{k} \mathrm{~d} y \\
& =-\int_{\mathbb{R}_{+}} \varphi(y) u\left(t_{k}, t_{k} y\right) \tilde{h}\left(t_{k}, y\right) \mathrm{d} y+\int_{\mathbb{R}_{+}} \phi(y) u_{x}\left(t_{k}, t_{k} y\right) \tilde{h}\left(t_{k}, y\right) t_{k} \mathrm{~d} y \\
& :=C_{k}+D_{k} .
\end{aligned}
$$


By (2.4), we get

$$
\begin{aligned}
C_{k}= & -\int_{\mathbb{R}_{+}} \varphi(y) u\left(t_{k}, t_{k} y\right) \tilde{h}\left(t_{k}, y\right) \mathrm{d} y \\
= & -\frac{1}{2} \int_{\mathbb{R}_{+}} \int_{\mathbb{R}_{+}} \varphi(y)\left(e^{-t_{k}|y-z|}-e^{-t_{k}|y+z|}\right) \tilde{h}\left(t_{k}, y\right) \tilde{h}\left(t_{k}, z\right) \mathrm{d} y \mathrm{~d} z \\
= & \left(-\frac{1}{2} \sum_{i=1}^{N} \iint_{\substack{|z-y|<\frac{\delta}{2} \\
y \in E_{i}, z \in \mathbb{R}_{+}}}-\frac{1}{2} \iint_{\substack{|z-y|<\frac{\delta}{2} \\
y \in E^{c}, z \in \mathbb{R}_{+}}}-\frac{1}{2} \iint_{|z-y|>\frac{\delta}{2}}\right) \\
& \times \varphi(y)\left(e^{-t_{k}|y-z|}-e^{-t_{k}|y+z|}\right) \tilde{h}\left(t_{k}, y\right) \tilde{h}\left(t_{k}, z\right) \mathrm{d} y \mathrm{~d} z:=C_{k 1}+C_{k 2}+C_{k 3} .
\end{aligned}
$$

Using (3.5) and (3.6), we obtain

$$
\begin{aligned}
\left|C_{k 1}\right| & \leq \sum_{i=1}^{N} \iint_{\substack{|y-z|<\frac{\delta}{2} \\
y \in E_{i}, z \in \mathbb{R}_{+}}}|\varphi(y)| \tilde{h}\left(t_{k}, y\right) \tilde{h}\left(t_{k}, z\right) \mathrm{d} y \mathrm{~d} z \leq \sum_{i=1}^{N} \iint_{\substack{y \in E_{i} \\
z \in F_{i}}}|\varphi(y)| \tilde{h}\left(t_{k}, y\right) \tilde{h}\left(t_{k}, z\right) \mathrm{d} y \mathrm{~d} z \\
& \leq \sum_{i=1}^{N}\left(\left|\varphi\left(\alpha_{i}\right)\right|+\varepsilon\right) m_{i}^{2}(1+\varepsilon)^{2} .
\end{aligned}
$$

By (3.36) and (3.7), we have

$$
\begin{aligned}
\left|C_{k 2}\right| & \leq \iint_{\substack{|z-y|<\frac{\delta}{2} \\
y \in E^{c}, z \in \mathbb{R}_{+}}}|\varphi(y)| \tilde{h}\left(t_{k}, y\right) \tilde{h}\left(t_{k}, z\right) \mathrm{d} y \mathrm{~d} z \leq\|\varphi\|_{L^{\infty}} \int_{y \in E^{c}} \tilde{h}\left(t_{k}, y\right) \mathrm{d} y \int_{\substack{|z-y|<\frac{\delta}{2} \\
z \in \mathbb{R}_{+}}} \tilde{h}\left(t_{k}, z\right) \mathrm{d} z \\
& \leq \varepsilon\|\varphi\|_{L^{\infty}}\left(\int_{y \in E^{c}} \tilde{h}\left(t_{k}, y\right) \mathrm{d} y\right) \leq \varepsilon\|\varphi\|_{L^{\infty}\left(\mathbb{R}_{+}\right)}\left\|h_{0}\right\|_{L^{1}\left(\mathbb{R}_{+}\right)} .
\end{aligned}
$$

Notice that, for $|y-z|>\frac{\delta}{2},|y+z|>\frac{\delta}{2}$, and using equation (3.36), we have

$$
\begin{aligned}
\left|C_{k 3}\right| & \leq \frac{1}{2} \iint_{|z-y|>\frac{\delta}{2}}|\varphi(y)| e^{-t_{k} \frac{\delta}{2}} \tilde{h}\left(t_{k}, y\right) \tilde{h}\left(t_{k}, z\right) \mathrm{d} y \mathrm{~d} z \\
& \leq \frac{1}{2} e^{-t_{k} \frac{\delta}{2}}\|\varphi\|_{L_{\infty}\left(\mathbb{R}_{+}\right)}\left\|h_{0}\right\|_{L^{1}\left(\mathbb{R}_{+}\right)^{\prime}}^{2}
\end{aligned}
$$

Using (2.5), we have

$$
D_{k}=\frac{1}{2} \int_{\mathbb{R}_{+}} \int_{\mathbb{R}_{+}} \phi(y) t_{k}\left[\operatorname{sgn}(z-y) e^{-t_{k}|z-y|}+e^{-t_{k}|z+y|}\right] \tilde{h}\left(t_{k}, z\right) \tilde{h}\left(t_{k}, y\right) \mathrm{d} z \mathrm{~d} y .
$$

As $D_{k}$ is symmetric in $y$ and $z$, there is a $\theta \in(0,1)$ such that

$$
\begin{aligned}
D_{k}= & \frac{1}{2} \int_{\mathbb{R}_{+}} \int_{\mathbb{R}_{+}} \phi(z) t_{k}\left[\operatorname{sgn}(y-z) e^{-t_{k}|y-z|}+e^{-t_{k}|y+z|}\right] \tilde{h}\left(t_{k}, z\right) \tilde{h}\left(t_{k}, y\right) \mathrm{d} z \mathrm{~d} y \\
= & \frac{1}{4} \int_{\mathbb{R}_{+}} \int_{\mathbb{R}_{+}}[\phi(y)-\phi(z)]\left[\operatorname{sgn}(z-y) e^{-t_{k}|z-y|}+e^{-t_{k}|z+y|}\right] \tilde{h}\left(t_{k}, y\right) \tilde{h}\left(t_{k}, z\right) \mathrm{d} y \mathrm{~d} z \\
= & \frac{1}{4} \int_{\mathbb{R}_{+}} \int_{\mathbb{R}_{+}} \varphi(y+\theta(z-y)) t_{k}(y-z)\left[\operatorname{sgn}(z-y) t_{k} e^{-t_{k}|z-y|}+e^{-t_{k}|z+y|}\right] \\
& \times \tilde{h}\left(t_{k}, y\right) \tilde{h}\left(t_{k}, z\right) \mathrm{d} y \mathrm{~d} z
\end{aligned}
$$




$$
\begin{aligned}
= & \left(\frac{1}{4} \sum_{i=1}^{N} \iint_{\substack{|y-z|<\frac{\delta}{2} \\
y \in E_{i}, z \in \mathbb{R}_{+}}}+\frac{1}{4} \iint_{\substack{y y-z \mid<\frac{\delta}{2} \\
y \in E^{c}, z \in \mathbb{R}_{+}}}+\frac{1}{4} \iint_{|y-z|>\frac{\delta}{2}}\right) \\
& \times \varphi(y+\theta(z-y)) t_{k}(y-z)\left[\operatorname{sgn}(z-y) e^{-t_{k}|z-y|}+e^{-t_{k}|z+y|}\right] \tilde{h}\left(t_{k}, y\right) \tilde{h}\left(t_{k}, z\right) \mathrm{d} y \mathrm{~d} z \\
:= & D_{k 1}+D_{k 2}+D_{k 3} .
\end{aligned}
$$

Using (3.5) and (3.6), get

$$
\begin{aligned}
\left|D_{k 1}\right| & \leq \frac{1}{2} \sum_{i=1}^{N}\left(\left|\varphi\left(\alpha_{i}\right)\right|+\varepsilon\right) \int_{y \in E_{i}} \tilde{h}\left(t_{k}, y\right) \mathrm{d} y \int_{z \in F_{i}} \tilde{h}\left(t_{k}, z\right) \mathrm{d} z \\
& \leq \frac{1}{2} \sum_{i=1}^{N}\left(\left|\varphi\left(\alpha_{i}\right)\right|+\varepsilon\right) m_{i}^{2}(1+\varepsilon)^{2} .
\end{aligned}
$$

As $\xi>0, \xi e^{-\xi} \leq 1$, from (3.36) and (3.7),

$$
\begin{aligned}
\left|D_{k 2}\right| \leq & \frac{1}{4}\|\varphi\|_{L^{\infty}\left(\mathbb{R}_{+}\right)} \iint_{\substack{|y-z|<\mid \frac{\delta}{2} \\
y \in E^{c}, z \in \mathbb{R}_{+}}} t_{k}|y-z| \mid \operatorname{sgn}(z-y) e^{-t_{k}|z-y|} \\
& +e^{-t_{k}|z+y|} \mid \tilde{h}\left(t_{k}, y\right) \tilde{h}\left(t_{k}, z\right) \mathrm{d} y \mathrm{~d} z \\
\leq & \frac{1}{2}\|\varphi\|_{L^{\infty}\left(\mathbb{R}_{+}\right)} \int_{y \in E_{c}} \tilde{h}\left(t_{k}, y\right)\left(\int_{z \in\left[y-\frac{\delta}{2}, y+\frac{\delta}{2}\right]} \tilde{h}\left(t_{k}, z\right) \mathrm{d} z\right) \mathrm{d} y \\
\leq & \frac{\varepsilon}{2}\|\varphi\|_{L^{\infty}\left(\mathbb{R}_{+}\right)} \int_{y \in \mathbb{R}_{+}} \tilde{h}\left(t_{k}, y\right) \mathrm{d} y \leq \frac{\varepsilon}{2}\|\varphi\|_{L^{\infty}\left(\mathbb{R}_{+}\right)}\left\|h_{0}\right\|_{L^{1}\left(\mathbb{R}_{+}\right)} .
\end{aligned}
$$

Notice that, for $\xi>0, \xi e^{-\xi}=\frac{\xi}{1+\xi+\xi^{2} / 2+\cdots} \leq \frac{\xi}{\xi^{2} / 2}=\frac{2}{\xi}$. Hence for $|y-z|>\frac{\delta}{2}$,

$$
\begin{aligned}
t_{k}|y-z| e^{-t_{k}|y-z|} \leq \frac{2}{t_{k}|y-z|}<\frac{4}{t_{k} \delta} & \\
\left|D_{k 3}\right| \leq & \frac{1}{4}\|\varphi\|_{L^{\infty}\left(\mathbb{R}_{+}\right)} \iint_{|y-z|>\frac{\delta}{2}} t_{k}|y-z| \mid \operatorname{sgn}(z-y) e^{-t_{k}|z-y|} \\
& +e^{-t_{k}|z+y|} \mid \tilde{h}\left(t_{k}, y\right) \tilde{h}\left(t_{k}, z\right) \mathrm{d} y \mathrm{~d} z \\
\leq & \frac{1}{2}\|\varphi\|_{L^{\infty}\left(\mathbb{R}_{+}\right)} \iint_{|y-z|>\frac{\delta}{2}} \frac{4}{t_{k} \delta} \tilde{h}\left(t_{k}, y\right) \tilde{h}\left(t_{k}, z\right) \mathrm{d} y \mathrm{~d} z \leq \frac{2\|\varphi\|_{L^{\infty}\left(\mathbb{R}_{+}\right)}}{t_{k} \delta}\left\|h_{0}\right\|_{L^{1}\left(\mathbb{R}_{+}\right)}^{2} .
\end{aligned}
$$

Step 3. From (3.10)-(3.19), we obtain

$$
\begin{aligned}
\left|B_{k}\right| \leq & \|\varphi\|_{L^{\infty}\left(\mathbb{R}_{+}\right)}\left\|h_{0}\right\|_{L^{1}\left(\mathbb{R}_{+}\right)}\left(\frac{3}{2} \varepsilon+\frac{1}{2} e^{-t_{k} \frac{\delta}{2}}\left\|h_{0}\right\|_{L^{1}\left(\mathbb{R}_{+}\right)}+\frac{2\left\|h_{0}\right\|_{L^{1}\left(\mathbb{R}_{+}\right)}}{t_{k} \delta}\right) \\
& +\frac{3}{2} \sum_{i=1}^{N}\left(\left|\varphi\left(\alpha_{i}\right)\right|+\varepsilon\right) m_{i}^{2}(1+\varepsilon)^{2} .
\end{aligned}
$$

Let $k \rightarrow \infty$, we have

$$
\limsup _{k \rightarrow \infty}\left|B_{k}\right| \leq \frac{3 \varepsilon}{2}\|\varphi\|_{L^{\infty}\left(\mathbb{R}_{+}\right)}\left\|h_{0}\right\|_{L^{1}\left(\mathbb{R}_{+}\right)}+\frac{3}{2} \sum_{i=1}^{N}\left(\left|\varphi\left(\alpha_{i}\right)\right|+\varepsilon\right) m_{i}^{2}(1+\varepsilon)^{2} .
$$

As $\varepsilon$ is arbitrary, the proposition is proved. 
Lemma 3.3 Let $\varphi \in C_{c}\left(\mathbb{R}_{+}\right)$, and $\phi \in C^{1}\left(\mathbb{R}_{+}\right)$be an antiderivative of $\varphi$, and

$$
\begin{aligned}
& A[t ; \varphi]=-\int_{\mathbb{R}_{+}} \phi(y) \frac{\partial}{\partial y}[y \tilde{h}(t, y)] \mathrm{d} y, \\
& B[t ; \varphi]=-\int_{\mathbb{R}_{+}} \phi(y) u(t, t y) \frac{\partial}{\partial y}[\tilde{h}(t, y)] \mathrm{d} y-2 \int_{\mathbb{R}_{+}} \phi(y) t u_{x}(t, t y) \tilde{h}(t, y) \mathrm{d} y .
\end{aligned}
$$

Then

$$
\limsup _{t \rightarrow \infty}(B[t ; \varphi]-A[t ; \varphi]) \geq 0
$$

Proof We divide the proof into three steps.

Step 1. Define $f(t):=\int_{\mathbb{R}} \phi(y) \tilde{h}(t, y) \mathrm{d} y$. Then from (3.1),

$$
\begin{aligned}
f^{\prime}(t)= & \int_{\mathbb{R}_{+}} \phi(y) \frac{\partial}{\partial t} \tilde{h}(t, y) \mathrm{d} y \\
= & \frac{1}{t} \int_{\mathbb{R}_{+}} \phi(y) \frac{\partial}{\partial y}[y \tilde{h}(t, y)] \mathrm{d} y \\
& -\frac{1}{t} \int_{\mathbb{R}_{+}} \phi(y) u(t, t y) \frac{\partial}{\partial y} \tilde{h}(t, y) \mathrm{d} y-\frac{1}{t} \int_{\mathbb{R}_{+}} 2 \phi(y) u_{x}(t, t y) \tilde{h}(t, y) t \mathrm{~d} y \\
= & -\frac{1}{t} A[t ; \varphi]+\frac{1}{t} B[t ; \varphi] .
\end{aligned}
$$

Integrating (3.21) from $t$ to $t^{2}$, we get

$$
f\left(t^{2}\right)-f(t)=\int_{t}^{t^{2}} \frac{B[s ; \psi]-A[s ; \psi]}{s} \mathrm{~d} s .
$$

Step 2. We claim that $Q:=\lim \sup (B[s ; \varphi]-A[s ; \varphi])<\infty$. Recall from Lemma 2.5 that there exists $d>\|u\|_{L^{\infty}\left(\mathbb{R}_{+} \times \mathbb{R}_{+}\right)}$such that, for all $t \geq 1$, supp $\tilde{h}(t, \cdot) \subset[0, d]$. From (3.36), we have

$$
\begin{aligned}
|A[t ; \varphi]| & =\left|-\int_{\mathbb{R}_{+}} \phi(y) \frac{\partial}{\partial y}[y \tilde{h}(t, y)] \mathrm{d} y\right|=\left|\int_{\mathbb{R}_{+}} \varphi(y) y \tilde{h}(t, y) \mathrm{d} y\right| \\
& \leq\|\varphi\|_{L^{\infty}\left(\mathbb{R}_{+}\right)} \int_{\text {supp } \tilde{h}(t, \cdot)}|y| \tilde{h}(t, y) \mathrm{d} y \leq\|\varphi\|_{L^{\infty}\left(\mathbb{R}_{+}\right)} d\left\|h_{0}\right\|_{L^{1}\left(\mathbb{R}_{+}\right)} .
\end{aligned}
$$

We claim that $B[t ; \varphi]$ is also bounded. To see this, write

$$
\begin{aligned}
B[t ; \varphi] & =-\int_{\mathbb{R}_{+}} \phi(y) u(t, t y) \frac{\partial}{\partial y}[\tilde{h}(t, y)] \mathrm{d} y-2 \int_{\mathbb{R}_{+}} \phi(y) t u_{x}(t, t y) \tilde{h}(t, y) \mathrm{d} y \\
& =-\int_{\mathbb{R}_{+}} \phi(y) \frac{\partial}{\partial y}[u(t, t y) \tilde{h}(t, y)] \mathrm{d} y-\int_{\mathbb{R}_{+}} \phi(y) t u_{x}(t, t y) \tilde{h}(t, y) \mathrm{d} y \\
& :=G_{1}+G_{2} .
\end{aligned}
$$

From (2.7) and (3.36), we have

$$
\left|G_{1}\right|=\left|\int_{\mathbb{R}_{+}} \varphi(y) u(t, t y) \tilde{h}(t, y) \mathrm{d} y\right| \leq\|\varphi\|_{L^{\infty}\left(\mathbb{R}_{+}\right)}\|u\|_{L^{\infty}\left(\mathbb{R}_{+} \times \mathbb{R}_{+}\right)}\left\|h_{0}\right\|_{L^{1}\left(\mathbb{R}_{+}\right)} .
$$


Using (2.5),

$$
\begin{aligned}
G_{2} & =-\frac{1}{2} \iint_{\mathbb{R}_{+} \times \mathbb{R}_{+}} \phi(y) t\left[\operatorname{sgn}(z-y) e^{-t|y-z|}+e^{-t|y+z|}\right] \tilde{h}(t, z) \tilde{h}(t, y) \mathrm{d} z \mathrm{~d} y \\
& =-\frac{1}{2}\left(\iint_{|y-z| \geq 1}+\iint_{|y-z|<1}\right) \phi(y) t\left[\operatorname{sgn}(z-y) e^{-t|y-z|}+e^{-t|y+z|}\right] \tilde{h}(t, z) \tilde{h}(t, y) \mathrm{d} z \mathrm{~d} y \\
& :=G_{21}+G_{22} .
\end{aligned}
$$

Noticing that, for $y, z \geq 0, t e^{-t|y+z|} \leq t e^{-t|y-z|} \leq \frac{t}{t|y-z|} \leq 1$, then

$$
\begin{aligned}
\left|G_{21}\right| \leq & \frac{1}{2} \iint_{[0, d]^{2}}|\phi(y)| t\left|\operatorname{sgn}(z-y) e^{-t|y-z|}+e^{-t|y+z|}\right| \tilde{h}(t, y) \tilde{h}(t, z) \mathrm{d} y \mathrm{~d} z \\
\leq & \|\phi\|_{L^{\infty}\left(\mathbb{R}_{+}\right)}\left\|h_{0}\right\|_{L^{1}\left(\mathbb{R}_{+}\right)}^{2} \leq\|\varphi\|_{L^{1}(\mathbb{R})}\left\|h_{0}\right\|_{L^{1}\left(\mathbb{R}_{+}\right)}^{2}, \\
G_{22}= & -\frac{1}{2} \iint_{|y-z|<1} \phi(y) t \operatorname{sgn}(z-y) e^{-t|y-z|} \tilde{h}(t, z) \tilde{h}(t, y) \mathrm{d} z \mathrm{~d} y \\
& +\left(-\frac{1}{2}\right) \iint_{|y-z|<1} \phi(y) t e^{-t|y+z|} \tilde{h}(t, z) \tilde{h}(t, y) \mathrm{d} z \mathrm{~d} y:=G_{221}+G_{222} .
\end{aligned}
$$

As $G_{221}$ is symmetric in $y$ and $z$, there is a $\theta \in(0,1)$ such that

$$
\begin{aligned}
\left|G_{221}\right| & \leq\left|\frac{1}{2} \iint_{|y-z|<1} \phi(z) t[\operatorname{sgn}(y-z)] e^{-t|y-z|} \tilde{h}(t, z) \tilde{h}(t, y) \mathrm{d} z \mathrm{~d} y\right| \\
& \leq\left|\frac{1}{4} \iint_{|y-z|<1}[\phi(y)-\phi(z)] t[\operatorname{sgn}(z-y)] e^{-t|y-z|} \tilde{h}(t, z) \tilde{h}(t, y) \mathrm{d} z \mathrm{~d} y\right| \\
& \leq \frac{1}{4} \iint_{|y-z|<1}|\varphi(z+\theta(y-z))| t|y-z| e^{-t|y-z|} \tilde{h}(t, z) \tilde{h}(t, y) \mathrm{d} z \mathrm{~d} y \\
& \leq \frac{1}{4}\|\varphi\|_{L^{\infty}\left(\mathbb{R}_{+}\right)}\left\|h_{0}\right\|_{L^{1}\left(\mathbb{R}_{+}\right)}^{2} .
\end{aligned}
$$

For $G_{222}$, choose the anti-derivative $\phi(y)=\int_{0}^{y} \varphi(s) \mathrm{d} s$. Noticing that $\|\phi\|_{L^{\infty}\left(\mathbb{R}_{+}\right)} \leq\|\varphi\|_{L^{1}\left(\mathbb{R}_{+}\right)}$,

$$
\begin{aligned}
\left|G_{222}\right| & =\left|\frac{1}{2} \int_{0}^{\infty} \phi(y) t e^{-t y} \tilde{h}(t, y)\left(\int_{|y-z|<1} e^{-t z} \tilde{h}(t, z) \mathrm{d} z\right) \mathrm{d} y\right| \\
& \leq \frac{1}{2}\left\|h_{0}\right\|_{L^{1}\left(\mathbb{R}_{+}\right)} \int_{0}^{\infty}|\phi(y)| t e^{-t y} \tilde{h}(t, y) \mathrm{d} y \\
& \leq \frac{1}{2}\left\|h_{0}\right\|_{L^{1}\left(\mathbb{R}_{+}\right)} \int_{0}^{\infty}\left|\frac{\phi(y)-\phi(0)}{y}\right| t y e^{-t y} \tilde{h}(t, y) \mathrm{d} y \\
& \leq \frac{1}{2}\left\|h_{0}\right\|_{L^{1}\left(\mathbb{R}_{+}\right)} \int_{0}^{d}|\varphi(\theta y)| \tilde{h}(t, y) \mathrm{d} y \leq \frac{1}{2}\left\|h_{0}\right\|_{L^{1}\left(\mathbb{R}_{+}\right)}^{2}\|\varphi\|_{L^{\infty}\left(\mathbb{R}_{+}\right)}
\end{aligned}
$$

where $\theta \in(0,1)$.

From (3.23)-(3.28), $|B[t ; \varphi]|$ is bounded in $t$. Together with (3.22), $-\infty<Q<\infty$.

Step 3. For each $\varepsilon>0$, there exists $K>0$ such that when $s>K, B[s ; \psi]-A[s ; \psi]<Q+\varepsilon$. Hence when $t^{2}>t>K$,

$$
f\left(t^{2}\right)-f(t)<\int_{t}^{t^{2}} \frac{Q+\varepsilon}{s} \mathrm{~d} s<(Q+\varepsilon) \log t,
$$


that is, $\frac{f\left(t^{2}\right)-f(t)}{\log t} \leq Q+\varepsilon$. For $r \in[1, \infty)$,

$$
f(r)=\int_{a}^{b} \phi(y) \tilde{h}(r, y) \mathrm{d} y \leq\|\phi\|_{C[a, b]}\left\|h_{0}\right\|_{L^{1}\left(\mathbb{R}_{+}\right)} \leq\|\varphi\|_{L^{1}(\mathbb{R})}\left\|h_{0}\right\|_{L^{1}\left(\mathbb{R}_{+}\right)}
$$

Hence

$$
0=\lim _{t \rightarrow \infty} \frac{f\left(t^{2}\right)-f(t)}{\log t} \leq Q+\varepsilon
$$

As $\varepsilon$ is arbitrary, Lemma 3.3 is proved.

Lemma 3.4 Suppose that $\widetilde{h}(t, \cdot)$ has a unique asymptotic density, that is, a $\mu \in \mathcal{M}[c, d]$ such that $\tilde{h}(t, \cdot) \rightarrow \mu$ as $t \rightarrow \infty$. Suppose $\mu=v+\sum_{i=1}^{\infty} m_{i} \delta_{\alpha_{i}}$ is the decomposition of $\mu$ into continuous and discrete parts (Lemma 2.6). Then, for all $\varphi \in C_{c}\left(\mathbb{R}_{+}\right), A[t ; \varphi] \rightarrow\langle y \mu, \varphi(y)\rangle$ as $t \rightarrow \infty$, and

$$
\mid\left\langle y \mu, \varphi(y)|| \leq \frac{3}{2} \sum_{i=1}^{\infty}\left|\varphi\left(\alpha_{i}\right)\right| m_{i}^{2}\right.
$$

Proof It is easy to see that

$$
A[t ; \varphi]=-\int_{\mathbb{R}_{+}} \phi(y) \frac{\partial}{\partial y}[y \tilde{h}(t, y)] \mathrm{d} y=\int_{\mathbb{R}_{+}} \varphi(y) y \tilde{h}(t, y) \mathrm{d} y \rightarrow\langle y \mu, \varphi(y)\rangle .
$$

From Proposition 3.2, we get

$$
\limsup _{k \rightarrow \infty}|B[t ; \varphi]| \leq \frac{3}{2} \sum_{i=1}^{\infty}\left|\varphi\left(\alpha_{i}\right)\right| m_{i}^{2}
$$

Together with (3.31) and Lemma 3.3,

$$
\begin{aligned}
\langle y \mu, \varphi(y)\rangle & =\lim _{t \rightarrow \infty} \int_{\mathbb{R}_{+}} \varphi(y) y \tilde{h}(t, y) \mathrm{d} y \\
& =\liminf _{t \rightarrow \infty} A[t ; \varphi] \leq \limsup _{t \rightarrow \infty} B[t ; \varphi] \leq \frac{3}{2} \sum_{i=1}^{\infty}\left|\varphi\left(\alpha_{i}\right)\right| m_{i}^{2} .
\end{aligned}
$$

Replacing $\varphi$ by $-\varphi$, we obtain (3.30).

Proof of Theorem 1.5 For (a), fix $i$, if $\alpha_{i}=0$, the conclusion holds. Assume $\alpha_{i}>0$, given $\varepsilon>0$, there exists a $\delta \in\left(0, \alpha_{i}\right)$ such that $\mu\left(\left[\alpha_{i}-\delta, \alpha_{i}+\delta\right]\right) \leq m_{i}+\varepsilon$.

If $\alpha_{j} \in\left[\alpha_{i}-\delta, \alpha_{i}+\delta\right], j \neq i, m_{i} \delta_{\alpha_{i}}+m_{j} \delta_{\alpha_{j}} \leq \mu$ implies that $m_{j} \leq \varepsilon$.

Let $\varphi \in C_{c}\left(\mathbb{R}_{+}\right), \varphi \geq 0$, supp $\varphi \subset\left(\alpha_{i}-\delta, \alpha_{i}+\delta\right) \subset \mathbb{R}_{+}, \max \varphi=\varphi\left(\alpha_{i}\right)$. From (3.30) and $\mu \geq 0$ we obtain

$$
m_{i} \alpha_{i} \varphi\left(\alpha_{i}\right)=\left\langle m_{i} \delta_{\alpha_{i}}, y \varphi(y)\right\rangle \leq\langle\mu, y \varphi(y)\rangle \leq \frac{3}{2}\left(m_{i}^{2} \varphi\left(\alpha_{i}\right)+\sum_{j \neq i} \varphi\left(\alpha_{j}\right) m_{j}^{2}\right) .
$$


If $\alpha_{j} \notin\left[\alpha_{i}-\delta, \alpha_{i}+\delta\right]$, then $\psi\left(\alpha_{j}\right)=0$. In any case,

$$
\varphi\left(\alpha_{j}\right) m_{j}^{2} \leq \varphi\left(\alpha_{j}\right) m_{j} \varepsilon \leq \varphi\left(\alpha_{i}\right) m_{j} \varepsilon
$$

Hence

$$
m_{i} \alpha_{i} \varphi\left(\alpha_{i}\right) \leq \frac{3}{2} \varphi\left(\alpha_{i}\right)\left[m_{i}^{2}+\varepsilon \sum_{j \neq i} m_{j}\right]
$$

Let $\varepsilon \rightarrow 0$, we get

$$
\alpha_{i} \leq \frac{3}{2} m_{i}
$$

For (b), from Lemma 2.5 and $\tilde{h}(t, \cdot) \rightarrow \mu$ we get supp $\mu \in[0, M]$. Together with $v \geq$ $0, m_{i}>0$, and (2.8), we obtain

$$
\operatorname{supp} v \subset[0, M], \quad \text { and } \quad \alpha_{i} \in[0, M]
$$

For (c), since $\mu=v+\Sigma_{i} m_{i} \delta_{\alpha_{i}}$ is a finite measure, $v \geq 0, m_{i}>0$ implies that

$$
\sum_{i} m_{i}<\infty
$$

Hence $m_{i} \rightarrow 0$, as $i \rightarrow \infty$, then from (3.32) we get

$$
\alpha_{i} \rightarrow 0, \quad \text { as } i \rightarrow \infty
$$

For (d), suppose $\mu$ does not have discrete parts over $(e, f) \subset \mathbb{R}_{+} \backslash\{0\}$, that is, no $\alpha_{i}$ is in $(e, f)$. Let $\varphi \in C_{c}(e, f)$, Then $\operatorname{supp} \varphi \cap\left\{\alpha_{1}, \alpha_{2}, \ldots\right\}=\emptyset$, from (3.30) we obtain $\langle\mu(y), y \varphi(y)\rangle=0$, hence $\left.y \mu\right|_{(e, f)}=0$, so $\left.\mu\right|_{(e, f)}=0$.

Notice that $\operatorname{supp} v \subset[0, M]$, as $\operatorname{supp} \mu \subset[0, M], v$ continuous and $\mu-v$ discrete. If $\alpha>0$, then it is not an accumulation point of $\left\{\alpha_{i}\right\}$. Hence there exists a $\delta \in(0, \alpha)$ such that $\bigcup_{i=1}^{\infty}\left\{\alpha_{i}\right\} \cap[(\alpha-\delta, \alpha+\delta) \backslash\{\alpha\}]=\emptyset$. Since $\left.\nu\right|_{(\alpha-\delta, \alpha) \cup(\alpha, \alpha+\delta)}=0$, as $v$ is a continuous measure, $\left.v\right|_{(\alpha-\delta, \alpha+\delta)}=0$. Hence $v=0$ in some neighborhood of each point of $(0, \infty)$, and hence $\left.v\right|_{\mathbb{R}_{+} \backslash\{0\}}=0$. As $v$ is a continuous measure, $v=0$, that is,

$$
\mu=\sum_{i=1}^{\infty} m_{i} \delta_{\alpha_{i}}
$$

We have

$$
\|\tilde{h}(t, \cdot)\|_{L^{1}\left(\mathbb{R}_{+}\right)}=\|h(t, \cdot)\|_{L^{1}\left(\mathbb{R}_{+}\right)}=\left\|h_{0}(\cdot)\right\|_{L^{1}\left(\mathbb{R}_{+}\right)} .
$$

Together with (3.35) and (3.36), we obtain

$$
\sum_{i=1}^{\infty} m_{i}=\sum_{i=1}^{\infty} m_{i} \delta_{\alpha_{i}}\left(\mathbb{R}_{+}\right)=\mu\left(\mathbb{R}_{+}\right)=\lim _{t \rightarrow \infty} \int_{\mathbb{R}_{+}} \tilde{h}(t, y) \mathrm{d} y=\left\|h_{0}\right\|_{L^{1}\left(\mathbb{R}_{+}\right)} .
$$


Competing interests

None of the authors have any competing interests as regards the manuscript.

\section{Authors' contributions}

All authors contributed equally and all authors approved the manuscript.

\section{Acknowledgements}

We thank the referees for the helpful comments and professor Tai-Man Tang for his help. The project is supported by the youth innovation fund projects of Tarim university President fund (TDZKQN201507).

Received: 6 August 2016 Accepted: 29 November 2016 Published online: 08 December 2016

\section{References}

1. Fokas, A, Fuchssteiner, B: Symplectic structures, their Bäcklund transformation and hereditary symmetries. Physica D 4, 47-66 (1981)

2. Constantin, A: On the scattering problem for the Camassa-Holm equation. Proc. R. Soc. Lond. A 457, 953-970 (2001)

3. Constantin, A, Gerdjikov, V, Ivanov, R: Inverse scattering transform for the Camassa-Holm equation. Inverse Probl. 22, 2197-2207 (2006)

4. Camassa, R, Holm, D, Hyman, M: A new integrable shallow water equation. Adv. Appl. Mech. 31, 1-33 (1994)

5. Coclite, GM, Karlsen, KH: A singular limit problem for conservation laws related to the Camassa-Holm shallow water equation. Commun. Partial Differ. Equ. 31, 1253-1272 (2006)

6. Coclite, GM, Ruvo, LD: A note on the convergence of the solutions of the Camassa-Holm equation to the entropy ones of a scalar conservation law. Discrete Contin. Dyn. Syst. 36, 2981-2990 (2016)

7. Constantin, A, Strauss, WA: Stability of peakons. Commun. Pure Appl. Math. 53, 603-610 (2000)

8. El Dika, K, Molinet, L: Stability of multipeakons. Ann. Inst. Henri Poincaré, Anal. Non Linéaire 26, 1517-1532 (2009)

9. El Dika, K, Molinet, L: Stability of multi-antipeakon-peakon profile. Discrete Contin. Dyn. Syst., Ser. B 12, 561-577 (2009)

10. Chen, GQ, Frid, H: Large-time behavior of entropy solutions of conservation laws. J. Differ. Equ. 152, 308-357 (1999)

11. Iftimie, D, Lopes, MC, Nussenzveig, HJ: Large time behaviour for vortex evolution in the half plane. Commun. Math. Phys. 237, 441-469 (2003)

12. Iftimie, D, Lopes, MC, Nussenzveig, HJ: On the large time behaviour of two-dimensional vortex dynamics. Physica D $179,153-160(2003)$

13. Iftimie, D: Large time behavior in perfect incompressible flows. In: Séminaires \& Congrés, vol. 15, pp. 119-179 (2007)

14. Jia, J, Kang, SG, Tang, TM: An asymptotic property of the Camassa-Holm equation. Nonlinear Anal., Theory Methods Appl. 97, 55-64 (2014)

15. Coclite, GM, Karlsen, KH: Periodic solutions of the Degasperis-Procesi equation: well-posedness and asymptotics. J. Funct. Anal. 268, 1053-1077 (2015)

16. Escher, J, Yin, Z: Initial boundary value problems of the Camassa-Holm equation. Commun. Partial Differ. Equ. 33, 377-395 (2008)

17. Hewitt, E, Stromberg, K: Real and Abstract Analysis. Springer, Berlin (1965)

\section{Submit your manuscript to a SpringerOpen ${ }^{\circ}$ journal and benefit from:}

- Convenient online submission

Rigorous peer review

- Immediate publication on acceptance

- Open access: articles freely available online

- High visibility within the field

- Retaining the copyright to your article 